\title{
DERIVING ACTIVITY THREAD IMPLEMENTATIONS FROM FORMAL DESCRIPTIONS USING TRANSITION REORDERING
}

\author{
Peter Langendörfer and Hartmut König \\ Brandenburg University of Technology at Cottbus \\ Department of Computer Science \\ PF 101344, D-03013 Cottbus \\ Germany \\ \{pl,koenig\}@informatik.tu-cottbus.de
}

\begin{abstract}
In this paper we show how formal descriptions can efficiently be mapped into activity thread implementations using transition reordering. This technique prevents semantic conflicts by reordering of statements at compile time. It considerably simplifies the derivation process and improves the efficiency of the generated code. The technique is implemented in the SDL compiler COCOS. We describe the approach as well as its implementation and show how semantic conflicts are resolved. Finally we present measurements which prove a considerable performance gain up to 120 per cent.
\end{abstract}

Keywords: Formal description techniques, SDL, automated protocol implementation, activity threads, transition reordering, configurable FDT compiler

\section{MOTIVATION}

The development of communication software is known to be an expensive and tedious process. Formal description techniques (FDTs) can help to considerably increase the quality of the protocols and telecommunication systems, and to shorten the time for their development. However, the benefits of FDTs are mainly used in the design, specification, performance prediction, verification, and testing phase. Automated implementation still represents a gap in this chain of development steps. Implementations are mostly carried out in traditional manner, i.e. man- 
ually. The main reason for this is that code automatically generated by an FDT compiler is mostly inadequate for applications in a real-life environment [Held95, Mans97]. It is more suited for prototyping rather than for final implementations. This shortage of automated protocol implementation cannot be compensated by other benefits such as a considerable reduction of the duration of the implementation process, independence of subjective implementation decisions, better conformance to the specification, and simplification of changes.

The inefficiency of automatically derived implementations is caused by the rigid implementation model, which determines the set of transformation rules, and by semantic constraints of the FDT. Further these compilers lack of means to adapt to a given implementation context, to efficiently handle the exchange of data packets, and to integrate several protocol layers. The semantics of the FDT can be simplly preserved by straight transforming it into the implementation. This mostly leads to a considerably performance overhead of the generated code caused by semantics-related computations and storing of data. Automatically generated implementations, however, will be only then accepted for practical applications if their efficiency comes close to manually coded implementations. Therefore, optimizations are required for the code generation process. In recent years several approaches were proposed to improve the efficiency of automated implementation techniques [Fisc95, Held95, Gotz96]. These approaches propose optimizations within the limits of the respective FDT semantics. The performance gain achieved is not adequate for final implementations. Other approaches such as a flexible runtime support system [Lang99] and the integration of efficient manual implementation techniques like activity threads [Henk97a, Henk97b] and integrated layer processing [Clar90, Abbo93, Brau96, Leue96] promise more optimistic results. The mapping strategies used in these techniques, however, do not permit it to straightly map the FDT semantics into the implementation. This is in part due to the integration of several layers which is usually not considered in formal descriptions. The compliance with the specification requires, therefore, to apply different transformation rules which can lead to conflicts with the FDT semantics as shown in [Henk97b].

In this paper we focus on the derivation of activity threads. In [Henk97a, Henk97b] we presented an approach to derive activity thread implementations from formal descriptions. As shown in these papers we could observe a considerably performance gain. We now present an improved approach for automatically deriving activity thread implementations. Unlike the approach presented in [Henk97a] it uses a new technique - the transition reordering - which allows it to handle almost 
all semantic conflicts at compile time. This simplifies the transformation procedure and remarkably improves the efficiency of the generated code. We present the approach for SDL [ITU93]. It can be also applied to other FDTs if the semantic constraints are taken into account as discussed in [Henk97b]. In Section 2, we introduce the activity thread implementation technique and discuss the semantic conflicts which may appear. Section 3 describes the transition reordering approach and its implementation in the SDL compiler COCOS. We also show how the semantic conflicts are resolved. In Section 4 we present measurements which show the effect of the approach. We further compare the received code with code generated by means of a commercial tool - the Cadvanced code generator of the SDT tool. In the concluding remarks we give an outlook on further research steps.

\section{MAPPING OF SDL SPECIFICATIONS ONTO ACTIVITY THREADS}

In this section we describe the principle of the transformation of SDL specifications onto activity threads as well as the possible semantic conflicts.

\subsection{MAPPING PRINCIPLE}

The activity thread technique [Clar85, Svob89] implements a protocol entity as a set of procedures where for each input event a corresponding procedure exists. The active elements in this model are the events. An incoming event activates the corresponding procedure which immediately handles the event and when producing an output calls the respective procedure of the next entity. The sequence of inputs and outputs (input $\rightarrow$ output $\rightarrow$ input ... input $\rightarrow$ output) results in a sequence of procedure calls called activity thread. Note that the term activity thread does not refer to an operating system thread. It denotes the execution path an event triggers in the protocol stack. Activity threads can run in both directions, upwards and downwards. The respective procedure calls are also denoted as upcalls and downcalls.

The activity thread technique provides very efficient implementations because it avoids the storing of events when calling the next protocol entity [Clar85]. Its semantic model (concurrent computation of different activity threads and synchronous communication), however, considerably differs from the semantic models of the standardized FDTs. The synchronous communication causes diverse semantic conflicts. They are discussed in the next subsection. Another constraint of this transformation is that spontaneous transitions cannot be handled [Henk97a, 
Henk97b]. They must be removed by refining the specification before code is generated. Therefore, FDT compilers usually apply the server model which supports a straight mapping of the FDT semantics instead of the more complicated activity thread approach.

The derivation approach of [Henk97a] separates the behaviour and data part of the SDL processes. It maps each process instance into a reentrant procedure and the data part into an instance control block. The events are the signals. Each output-statement is mapped on a procedure call. The delivery of the signals is organized via an additional loop construction which forms the frame of the implementation. This loop construction is mapped on an activity thread scheduler which handles concurrent activity threads, the delivery of the signals and semantic conflicts. Details of this mapping strategy can be found in the referenced papers (see also Section 3.1).

\section{$2.2 \quad$ SEMANTIC CONFLICTS}

The semantic conflicts which may appear when directly mapping SDL specifications onto activity threads can be divided in two groups: interferences of transitions and overtaking of signals. In both cases they are caused by the appearance of cyclic process call sequences at runtime.

Interference of transitions. In the communication between two or more processes the activity thread implementation principle may cause situations in which a signal is consumed by the receiving process before the sender has finished its transition. This may lead to the following errors:

- discarded signals, and

- inverted order of state changes and variable assignments.

The discarding of signals may occur when a procedure call implementing an output -statement is executed before the assignment of next state. In this case, the process instance cannot change its state until the called procedure has returned control. Figure 1.1 gives an example for such a situation.

An implementation that is conform to the specification has to guarantee that process $P 1$ changes to state update after sending the signal auth. Then it can accept the signals account or reject by means of which process P2 responds to auth. In contrast to the expected correct behaviour, the signals account and reject respectively, are always discarded by process $P 1$, because $P 1$ remains in state login until the procedure call 


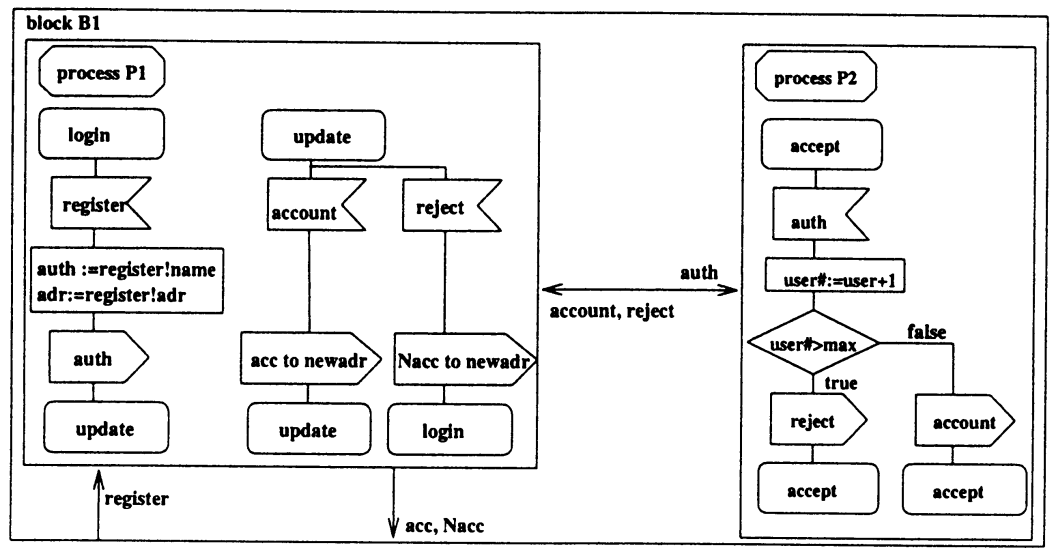

Figure 1.1 Example for discarding of a signal

for the output of auth is finished. Figure 1.2 shows the resulting cyclic process call sequence.

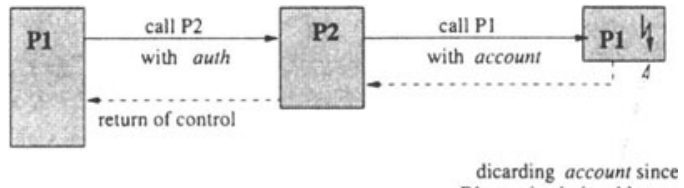

P1 remains in its old state

Figure 1.2 Process call sequence for example of figure 1.1

Overtaking of signals. SDL guarantees that the order of signals sent via a signal route or a channel is preserved at destination. If, for instance, a signal $s 1$ is sent at time $t$ and a signal $s 2$ at time $t+x$, then $s 1$ has to be delivered before 52 . When applying the activity thread approach signals may overtake each other.

This situation may happen if a transition contains several outputstatements. The mapping of output-statements onto procedure calls causes that the second, third and any further procedure call is delayed until the first one and all successor calls have been finished. If one of these successors uses a signal route that is also used by one of the other output-statements of the transition with multiple outputs, the signals overtake. Figure 1.3 shows an SDL specification in which the preconditions for signal overtaking are fulfilled. 


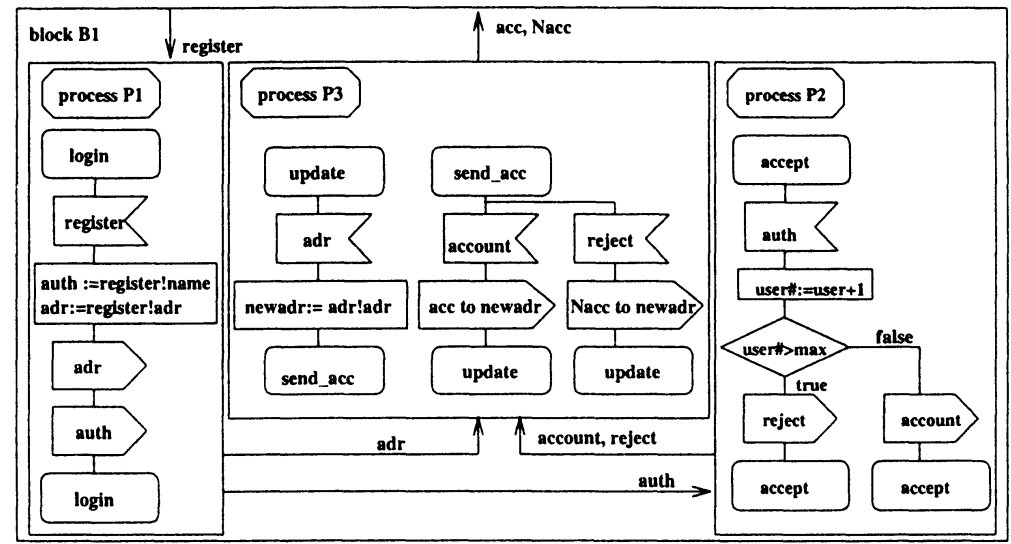

Figure 1.3 SDL System with cycles and the possibility of signal overtaking

In a straight forward generated activity thread implementation of the specification given in figure 1.3 the signal register would trigger the following execution. Process $P 1$ sends signal auth to process $P 2$ that replies with reject or account. $P 1$ immediately forwards these signals to process $P 3$ which responds with acc or Nacc to the address given in newadr. After that the control returns to $P 1$ which sends the signal $a d r$ to $P 3$. In contrast to the specified behaviour, one of the signals account or reject now has overtaken adr. As consequence, P3 sends the signal acc or Nacc to a wrong address. The resulting process call sequence is depicted in figure 1.4 .

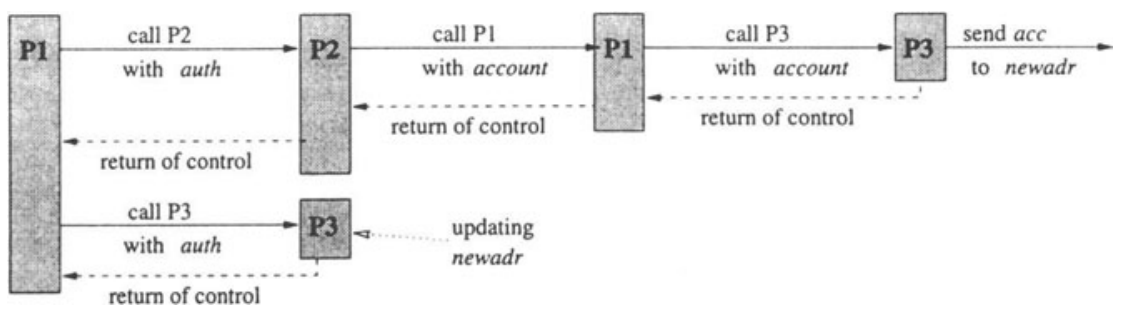

Figure 1.4 Process call sequence for example of figure 1.3 


\section{TRANSITION REORDERING 3.1 PRINCIPLE}

In [Henk97a] the semantic conflicts discussed in section 2 are resolved at runtime. The solution consists in delaying the procedure calls for the output-statements until the end of the transition. This is done by the procedure represented in figure $1.5 \mathrm{a}$. When executing an outputstatement the signal and its receiver are stored in a signal list. The activity thread scheduler processes the signal list in a FIFO manner. It always activates the receiver process of the first entry. The receiver process consumes the signal and executes the respective transition. By this, further signals can be added by means of an append function to the signal list if the transition contains output-statements. After finishing the procedure the control is returned to the scheduler. The shortage of this mechanism is that the execution of the output-statement is delayed, i.e. it may be processed after output-statements of other transitions. This leads to an asynchronous communication and considerably increases the overhead of an activity-thread implementation.
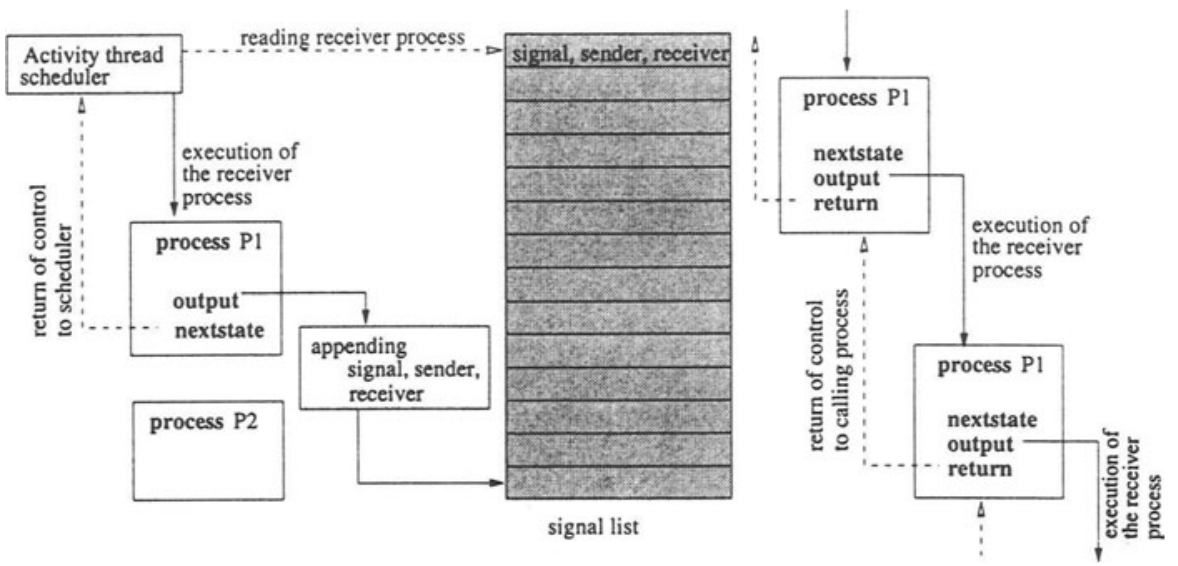

Figure 1.5 Mapping on activity threads

The transition reordering designates that the SDL statements of a transition are not implemented in the order as they are specified. They are reordered at compiler time in such a way that the semantic conflicts described above cannot occur. The approach adapts the principle 
of code restruction which is applied for loop optimization in modern programming language compilers [Aho96].

The reordering only concerns the output-statements. They are implemented in two steps:

1. Replace the output-statement by an operation which stores the signal.

2. Append the output-statement with the stored signal to the end of the transition. If a decision-statement follows the output-statement the modified output-statement has to be appended to each branch of the decision-statement.

All other SDL statements are implemented in the order as they are specified, i.e. only the sending of the signals is delayed. This approach does not require any additional runtime support. The output-statements are only executed when the transition has reached the successor state.

Thus, according to the activity thread principle the receiver process is triggered by the sender process. The control only returns to the receiver process if no further output-statements have to be processed or if in connection with a server model implementation a signal is sent to an asynchronous (buffering) interface (see figure 1.5b).

Using transition reordering the indirect realization of the activity thread approach using a special loop construction and runtime support features as proposed in [Henk97a] can be avoided except some special cases (see below). The transitions can straight be mapped on procedures, i.e. a direct derivation of activity threads is possible by means of transition reordering.

The reordering of actions inside a transition is allowed, because:

1. SDL processes are nonpreemptive. A transition is finished before a new transition can be executed.

2. Signals cannot be modified after output.

These conditions guarantee that the shift of the output-statements to the end of the transition do not influence the compliance between specification and implementation. Condition (1) expresses that the outputstatements are also executed if they are implemented at the end of the transition, because there is no statement in SDL which can interrupt the execution of the transition. Condition (2) ensures that the data transferred by a signal cannot be modified by a statement following the output-statement. Thus, the delay in the sending of the signals cannot lead to a transmission of falsified data.

In the next subsections we show how the semantic conflicts discussed above can be avoided by applying transition reordering. 


\subsection{AVOIDING TRANSITION INTERFERENCES}

The reordering of transitions ensures that assignments to variables and state changes are carried out in the correct order even if cyclic process call sequences appear. It prevents the discarding of signals and the inversion of variable settings. The conformance between specification and implementation is preserved. Figure 1.6 shows the specification of the example from figure 1.1 after transition reordering.

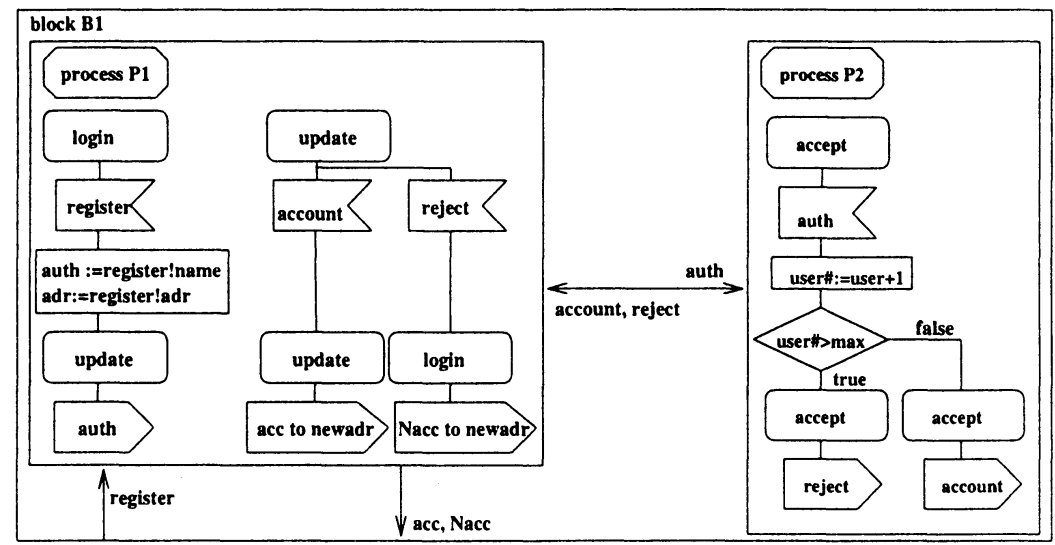

Figure 1.6 Example of figure 1.1 after transition reordering

Figure 1.7 depicts the respective process call sequence and indicates that the implementation is executed correctly.

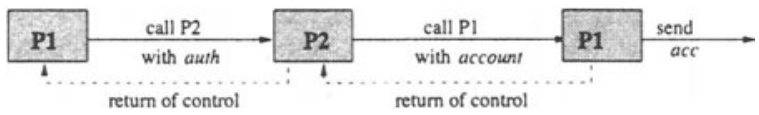

Figure 1.7 Process call sequence for example of figure 1.6

\subsection{AVOIDING OVERTAKING OF SIGNALS}

Since at compile time the structure of the SDL system as well as the logical structure of the implementation are known the compiler can determine whether there are transitions with multiple outputs which lead to an overtaking of signals. This is done by analysing the execution pathes and the state changes of transitions as described below. Based 
on this information it can determine in which order the procedure calls implementing output-statements have to be inserted into the final code to prevent overtaking of signals.

In the first step a data flow analysis is applied to detect possible cyclic process call sequences. For this purpose, for each signal sent in a transition with several output-statements the set of successor signals and of receiving processes is determined. The algorithm is recursively applied to the successor signals and terminates when the signals are either sent to the environment or to a process instance implemented according to the server model. For this analysis, it is assumed that each receiving process is in a state where it can accept the incoming signal and execute the corresponding transition. Thus, we get the execution path triggered by each output-statement. If possible cyclic behaviour is detected it is proved whether the first and the last transition of the call sequence use the same signal route. If this happens the following cases have to be distinguished:

1. the signal route is used for multiple output in the same transition,

2. the signal route is used by different transitions.

Case (1) allows it to implement the output-statements in the same order as they are specified. No additional measures have to be taken. In case (2) the output-statement triggering the cyclic process call sequence has to be executed last so that the signal which may be overtaken is sent before the other signals. These output-statements are appended to the end of the transition code.

Figure 1.8 shows the order in which the example given in figure 1.3 is implemented after reordering of the transitions.

Note that the outputs of process $P 1$ are not only delayed but that their order has also been changed. Figure 1.9 shows again the resulting process call sequence. In this implementation the signals $a d r$ and account arrive in the correct order at $P 3$.

There are cases in which the transition reordering does not resolve the semantic conflicts. e.g. for multiple outputs on the same signal route or for cyclic process call sequences which lead to the same transition. In these cases the runtime solution proposed in [Henk97a] has to be applied as before.

\subsection{IMPLEMENTATION OF THE TECHNIQUE}

The transition reordering approach as described in Section 3.1 has been implemented for the activity thread technique in the SDL compiler 


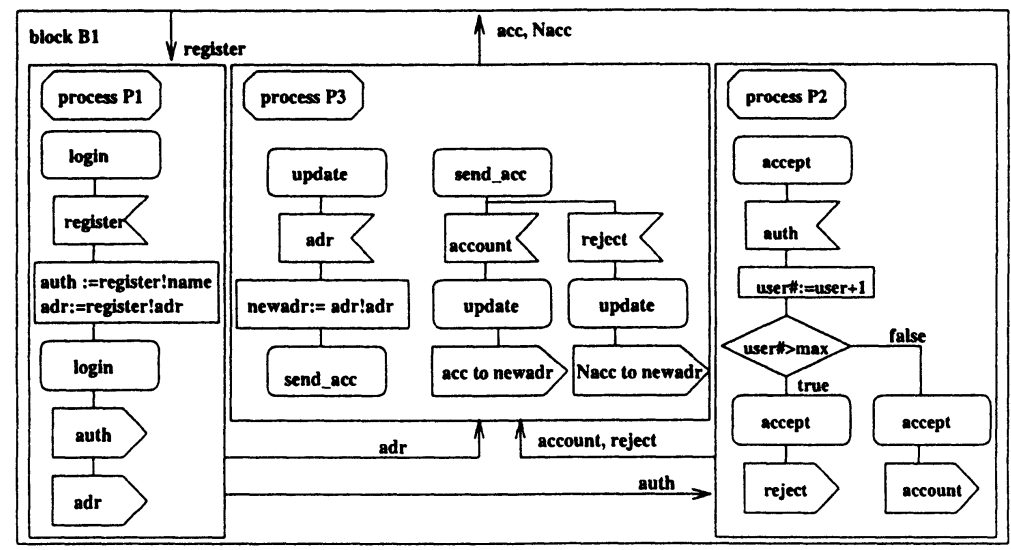

Figure 1.8 Figure 1.3 after transition reordering

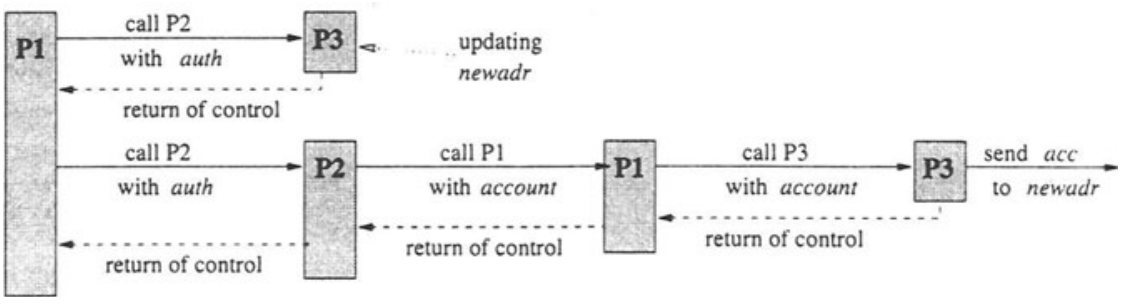

Figure 1.9 Process call sequence for example of figure 1.8

COCOS (Configurable Compiler for SDL) [Lang99]. COCOS is a configurable compiler that supports different implementation strategies. Currently these are the server model and the activity thread model. The integrated layer processing will be added soon. Another feature of COCOS is its ability to adjust to the given implementation context. This feature overcomes one of the main shortages of automated implementation: the rigid implementation model. Depending of the selected implementation strategy a tailored runtime system is generated which optimally adapts to the implementation environment. The configuration process is controlled by implementation-oriented annotation called $i S D L$ which beside the selection of the mapping strategy integrates implementationoriented information such as number of processors and kind of memory organization into the code generation process. A detailed description of COCOS and iSDL is contained in [Lang99]. 


\section{PERFORMANCE MEASUREMENTS}

In this section we describe measurements which demonstrate the effect of the approach. Besides measurements of activity thread implementations with and without transition reordering we also present measurements which compare the efficiency of these implementations with code generated by a commercially available tool - the Cadvanced code generator of the SDT tool version 3.4 [Tele98]. For the measurements, we used the SDL specification of a client/server application based on a TCP/IP protocol stack described in [Hint97]. The structure of the specification is depicted in figure 1.10 .

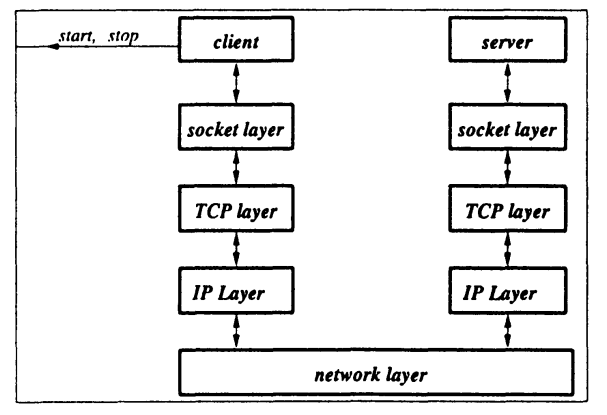

Figure 1.10 Structure of the applied SDL specification

The client process generates data which have to be transmitted to the server process and confirmed. Before the client can open a TCP connection it has to ask for a socket. The server process initiates a passive open to the socket layer. Then it listens. The socket process forwards the application data to the TCP process and vice versa. The TCP process contains the known functionality of the protocol: division of the application data into TCP segments, timer control of the transmission, discarding of duplicate IP packets, flow control, congestion control and error handling. The IP process has a simplified functionality in this specification. It only supplements the IP headers to TCP packets and assigns the incoming IP packets to TCP connections. Fragmentation is not included. The network process simulates the network and transfers the packets from the client to the server site.

We measured the time for the transmission of a sequence of packets starting from the transmission of the first packet until the reception of the last acknowledgement at client site. The process client sends the signal start to the environment when it forwards the first packet. The end of the transmission is indicated by the stop signal. 
COCOS as well as Cadvanced implement the whole specification by a single operating system process. Thus, the interfaces between the generated code and the operating system do not influence the measurement results. We generated two activity thread implementations with COCOS: one without transition reordering and the other with applying this technique. In these implementations the processes socket, TCP, and IP were mapped on activity threads (as described above), while the processes client, server, and network were implemented according to the server model. (This mapping corresponds to the usually applied integration of activity thread implementations into the execution environment, because activity thread implementations require an asynchronous interface at the bottom and the top of the protocol stack.) The generated $\mathrm{C}$ code of all implementations was compiled with the gcc compiler without any optimizing options. The measurements were made on a Sun Sparc 20 workstation with four processors and Solaris 2.5 as operating system. We measured the time for the transmission of 1000,10000 an 20000 packets with a packet size of 1024 byte. Each measurement was repeated 50 times. To exclude exceptional behaviour extreme good and extreme bad measured values were eliminated and then the arithmetic middle of the remaining measured values was calculated. The results are given in figure 1.11 .
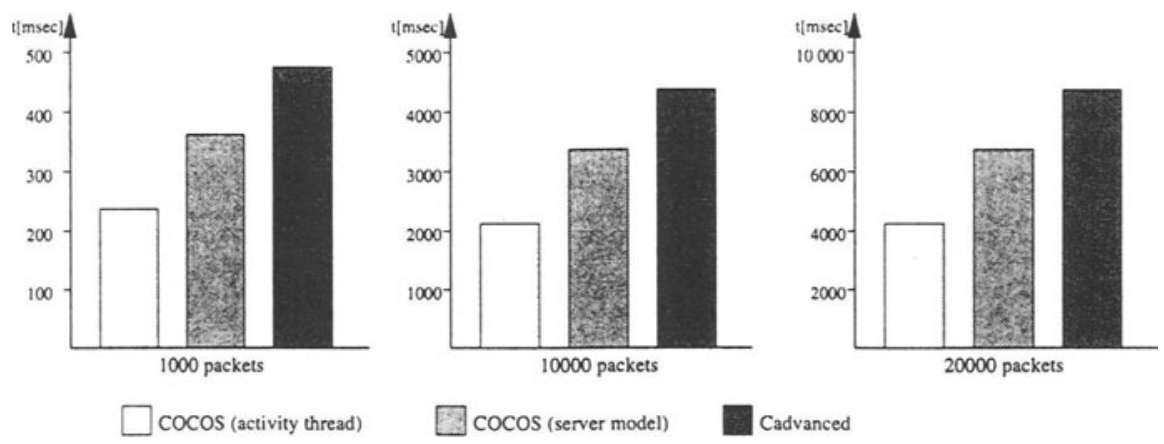

Figure 1.11 Measurements of different implementation strategies

The results show that activity thread implementations with transition reordering achieve a remarkable better performance (between 25 - 30 per cent) than those which apply the approach of [Henk97a]. This increase can be explained by the optimized mapping strategy which resolves semantic conflicts at compile time. 
The comparison with the Cadvanced code generator shows that both activity thread implementations achieved an about 60 and 120 per cent, respectively, better performance. This proves that by using the activity thread technique a considerably better performance can be obtained. Note that both compilers use the same technique to reduce the operating system overhead. For each signal that is sent memory has to be allocated to store its data. This memory is usually allocated by the operating system at runtime. Cadvanced [Tele98] as well as COCOS allocate this memory during system initialization.

Further performance improvements are expected the avoidance of data copy operations [Lang99] and an optimized timer management.

\section{CONCLUDING REMARKS}

In this paper, we have presented with the transition reordering an approach which allows it to efficiently map formal descriptions in an EFSM-based FDT onto activity thread implementations. The transition reordering resolves almost entirely semantic conflicts at compile time. Additional runtime support is only needed for some exceptional cases. The approach has been integrated in the configurable SDL compiler COCOS. The measurements we have presented indicate a considerable increase in the efficiency of the generated code. For the application of the transition reordering, an increase of about 25 to 30 per cent was measured. Compared with the code generated by a commercially available tool like the Cadvanced compiler an increase up to 120 per cent could be observed. Further improvements are expected by optimizations such as avoidance of data copy operations and a more efficient timer management which are step by step introduced into the COCOS compiler. The considerable progress achieved and the mentioned further optimizations show that there is still a large potential for improving the efficiency of automated code generation.

Currently we are introducing the integrated layer processing as third implementation strategy in the configurable compiler. Thereafter, we plan to evaluate our tool and the different implementation techniques by comparing it with hand-coded implementations of the TCP/IP protocol stack or other appropriate protocols. The latter task seems simple, but there scarcely exist real-life protocol implementations of the TCP/IP protocol stack or other protocols which are derived from a formal description and thus could be used as a basis for such a comparison. For TCP/IP, there is the additional problem that most real-life implementations are kernel-integrated implementations. The derivation of such kind of implementations is not the objective of our current research. 
TCP/IP implementations though will not be the main application area of automated protocol implementation techniques. We see the potential application of automated implementation techniques more in the area of application protocols and especially for newly designed protocols to fast derive an implementation from the formal description which can be optimized if needed. This will help to close the "implementation gap" in the protocol development process mentioned in the introduction and allow a continuous protocol development based on formal descriptions from the design via automated code generation to testing.

\section{References}

[Abbo93] Abbott M.; Peterson, L.: Increasing network throughput by integrating protocol layers. IEEE/ACM Transaction On Networking 1, October 1993, pp. 600-610.

[Aho96] Aho, A. V.; Sethi, R.; Ullman, J. D.: Compilers: principles, techniques, and tools. Addison Wesley, 1996.

[Brau96] Braun, T.; Chrisment, I.; Diot, C.; Gagnon, F.; Gautier, L.; Hoschka, P.: ALFred, an ALF/ILP Protocol Compiler for Distributed Application Automated Design. Rapport de Recherche Nr. 2786, INRIA Jan. 1996.

[Clar85] Clark, D. D.: The structuring of systems using upcalls. Proc. 10 th ACM SIGOPS Symp. Oper. Syst. Principles, 1985, pp. 171-180.

[Clar90] Clark, D. D.; Tennenhouse, D. L.: Architectural considerations for a new generation of protocols. ACM SIGCOMM, 1990, pp. 200-208 .

[Fisc95] Fischer, S.; Effelsberg, W.: Efficient Configuration of Protocol Software for Multiprocessors. In Puigjaner, R. (ed.): High Performance Networking VI, Chapman \& Hall, 1995, pp. 195210.

[Gotz96] Gotzhein, R.; Bredereke, J.; Effelsberg, W.; Fischer, S.; Held, T.; Koenig, H.: Improving the Efficiency of Automated Protocol Implementation Using Estelle. Computer Communications 19 (1996), pp. 1226-1235.

[Held95] Held T.; König, H.: Increasing the Efficiency of Computeraided Protocol Implementations. In Vuong, S., Chanson, S. (eds.): Protocol Specification, Testing and Verification XIV, Chapman \& Hall, 1995, pp. 387-394.

[Henk97a] Henke, R.; König, H.; Mitschele-Thiel, A.: Derivationof Efficient Implementations from SDL Specifications Employing 
Data Referencing, Integrated Packet Framing and Activity Threads. In Cavalli, A.; Sarma, A. (eds.): SDL'97 Time for Testing. Elsevier, 1997, pp. 397-414.

[Henk97b] Henke, R.; Mitschele-Thiel, A.; Koenig, H.: On the Influence of Semantic Constraints on the Code Generation from Estelle Specifications. In Mizuno, T. et al. (eds.): Formal Description Techniques and Protocol Specification, Testing and Verification (FORTE X/PSTV XVII'97), Chapman \& Hall, 1997, 399-414.

[Hint97] Hintelmann, J.; Westerfeld, R.: Performance Analysis of TCP's Flow Control Mechanisms Using Queueing SDL. In Cavalli, A.; Sarma, A. (eds.): SDL'97 Time for Testing. Elsevier, 1997, pp. 69-84.

[ITU93] ITU-T. Z.100: Specification and Description Language (SDL). ITU, 1993.

[Lang99] Langendörfer, P.; König, H.: COCOS - A Configurable SDL Compiler for Generating Efficient Protocol Implementations. In Proceedings 9th SDL Forum, Elsevier, 1999.

[Leue96] Leue, S.; Oechslin, P.: On Parallelizing and Optimizing the Implementation of Communication Protocols. IEEE/ACM Trans. on Networking, 4 (1), 1996.

[Mans97] Mansurov, N.; Chernov, A.; Ragozin A.: Industrial Strength Code Generation from SDL. In Cavalli, A.; Sarma, A. (eds.): SDL'97 Time for Testing. Elsevier, 1997, pp. 415-430.

[Svob89] Svobodova, L.: Implementing OSI Systems. IEEE Journal on Selected Areas in Communications, 7(7),1989, pp. 1115-1130.

[Tele98] Telelogic Malmö AB: SDT 3.2 User's Guide. SDT 3.2 Reference Manual. 1998. 